\title{
Dientes ChiquiTICOS: an analysis of juvenile dentition and dental health in Costa Rican indigenous communities
}

\author{
Alfredo García*1, Christina Guzzo2
}

\begin{abstract}
This study surveyed the dental health of three Costa Rican indigenous populations and two rural, non-indigenous communities. Sixty-six individuals, both children and adults, were interviewed regarding dental hygiene practices and the dentition of eightyeight children from the ages of two to thirteen was examined. The indigenous populations, on average, showed a more important number of anterior dental pathologies as compared to a non-indigenous group (42\% vs $20 \%)$. Collectively, both access to and utilization of dental healthcare were worse within the indigenous communities; however, there was still great variation amongst all five sites.
\end{abstract}

KEYWORDS: dentition, caries, dental health, hygiene, Costa Rica, idigenous.

\section{INTRODUCTION}

Costa Rica, with a total land area of 51,100 square kilometers, is a country slightly smaller than the state of West Virginia and has a total population of 4.02 million (1). The indigenous population, residing on twenty-two indigenous reserves, comprises only $1.7 \%$ of this population. There are currently six indigenous tribes in Costa Rica: Boruca, Bribri, Cabécar, Guaymi, Maleku, and Térraba. Three of these groups are discussed in this report: the Borucas, the Malekus, and the Bribris (Figure 1).

The Boruca population consists of approximately 2,000 individuals and is located in the province of Puntarenas in the southeastern section of Costa Rica. This study contains data collected at the community of Boruca, which is the largest of the three reserves. Due to construction of the Interamerican Highway, which

*To whom correspondence should be addressed: Alfredo Garcia Department of Biological Anthropology and Human Anatomy, Duke University, Box 95797, Durham, North Carolina

Email: Alfredo.garcia@duke.edu

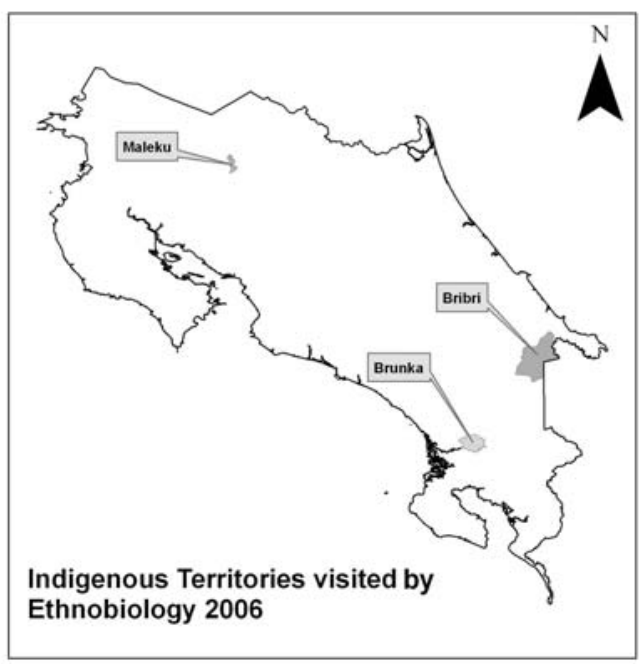

Figure 1. Location of indigenous territories within Costa Rica

now runs though the town of Rey Curré, transportation to and from the community has become much easier. Consequently, access to medical services has improved. 
The Maleku are a group of roughly 500 individuals found in the province of Alajuela in Northern Costa Rica. The community at La Fortuna, which is the southernmost group of Malekus, is divided into the three different villages; Palenque El Sol, Palenque Tonjibe, and Palenque Margarita (2). This study contains data collected at the villages of Palenque Tonjibe and Palenque Margarita.

The Bribri of Talamanca is the largest of all Costa Rican indigenous reservations, spanning a territory of 43,690 hectares (3). For this study, two communities within this Bribri reserve were visited: Kachabri and Shiroles. Preservation of the Bribri culture can be seen in the maintenance of their language and in the continued use of awapa (shamans) for healing purposes. The Bribri health services have been characterized by state medical authorities as deficient in many aspects. The government has recognized the lack of attention, absence of house visits, difficulties regarding accessibility, and nutrition programs which are not meeting expectations (3).

In addition to the three indigenous reserves, two nonindigenous communities were visited to serve as comparison groups: La Gamba and Cahuita. La Gamba is a sustainable farming community located in the province of Puntarenas. It was established in 1978 as a bananal (banana plantation) and has since become an example of organic and subsistence farming practices. Cahuita is an Afro-Caribbean community located in the province of Limón along the east coast of Costa Rica. With the lure of Cahuita National Park and the region's deep historical roots, this community has become a well-known tourist destination. These sites were chosen because of the two different, yet common, Costa Rican communities that they represent. La Gamba gives information on the rural population devoted to cultivating land (known as the campesino culture). Cahuita gives insight into areas that are dominated by tourist influence and activity.

Collectively, due to their location in remote and inhospitable rural areas, the indigenous communities often lack access to health care, schools, electricity, and potable water. Socialized healthcare has reached the indigenous communities of Costa Rica only within the past decade. In 1999, the government completed distribution of identification cards to all indigenous people through the National Indigenous Commission (4). Consequently, all indigenous people were given the benefits of citizenship including seguro social (socialized health insurance). While this helped to facilitate access to public medical facilities for all citizens, the question remains as to how this has affected indigenous communities, in particular, their utilization of dental healthcare.
Some work has been done on the dental health of the Costa Rican indigenous populations, but nothing as extensive as this report. Statistical figures do exist illustrating the number of dental visits (5); however no real field work has been conducted by health specialists concerning these indigenous communities. Duke undergraduate Shaina Wahl of the 2005 Organization for Tropical Studies Field Ethnobiology program examined the dentition of the Boruca and Maleku communities, but difficulties in her investigation necessitated further research. Her sample size spanned from individuals 3 to 20 years of age, a range that incorrectly included both deciduous and permanent dentition, with her method of analysis consisting of simply counting the number of caries lesions present in the mouth. However, when evaluating the number of cavities present, it is essential to distinguish between deciduous (primary) and succedaneous (permanent) dentition; the two are considerably different anatomical entities (6). Some of these differences between the two types of dentition, such as enamel thickness, contribute to the appearance of more dental pathologies in deciduous dentition than in permanent dentition. Wahl's (7) discussion also failed to explore the underlying principles behind the answers she received and the severity of dental pathologies encountered.

In contrast, this study aims to fully and adequately represent the oral health of three indigenous communities by employing a series of more systematic approaches. A complete dental inventory was conducted using an age range that encompassed all the years of primary dentition up until the age in which the full permanent dental arcade develops. The severities of pathologies, not just the quantity of pathologies, were also analyzed. This study also includes the results of extensive interviews with children and adults on the dental hygiene of both indigenous and non-indigenous communities. Factors which influence indigenous children's adherence to self-care standards were appraised through a survey of access to and utilization of healthcare, pervasiveness of health education, attitudes towards self-care standards, and identifiable cultural factors. The results of this study can serve to educate indigenous groups on how to improve dental hygiene, utilization of healthcare, and ultimately the overall health status of their children.

\section{MATERIALS AND METHODS}

Research was conducted at the indigenous reserves of the Boruca, Maleku, and Bribri, the Afro-Caribbean community of Cahuita, and the campesino community of La Gamba. Community consent was obtained by the Organization for Tropical Studies (OTS) prior to arriving at these locations and verbal informed consent 
was sought and obtained from each consultant prior to questioning. In the case of children, consent was received from their guardian before both dental examinations and general questioning. Consultants were chosen at random. A day was spent at each of the locations in which the researchers walked around the community and met with children in homes or schoolhouses (Figure 2). Each interview was conducted with both authors present.

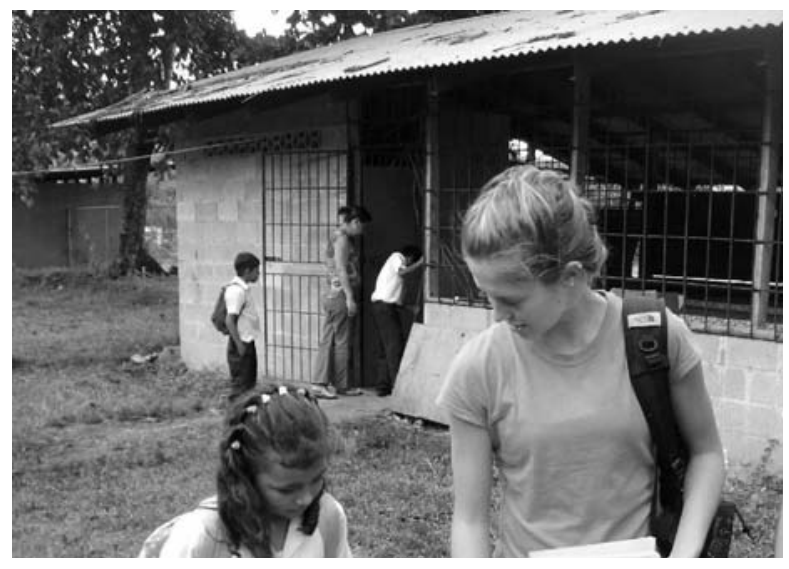

Figure 2. Dental hygiene interview

Notes were made while looking into the open mouths of children and dental atrophies were recorded on standardized worksheets with as much detail as possible. The quality of dental health of each child was quantified using a system devised by the authors. One gradation point was given for each of the following dental atrophies: (1) pain and/or bleeding; (2) open mesial interstitial caries on central incisors (given only one gradation point for both incisors); (3) other anterior caries (each given a separate gradation point); (4) medium to very large untreated caries; (5) untreated caries on the permanent dentition; and (6) considerable plaque formation (given only one gradation point for overall appearance). Filled or treated cavities were not given a gradation point since these pathologies had been successfully addressed by a dentist.

Thorough semi-structured interviews were carried out with fourteen children at each community, seven of each gender. The guardian of each child was also interviewed. In addition, interviews were conducted with directors of the secondary schools, healthcare workers, elders, and parents of children too young to be interviewed directly (7). Both closed and open-ended questions were used for gathering dental data, using a list of thirty-five questions as a guide. Responses were recorded using notebooks and microcassette recorders, if given permission from the consultant (Figure 3). General observations were also recorded in hand notebooks, and digital cameras were utilized to aid with visual observations. Upon completion of each consultation, either a collection of toothbrushes, toothpastes, and dental educational materials or cookies were given to all children who contributed to our dataset. Adults were compensated for their time with dental health pamphlets or small gifts of food.

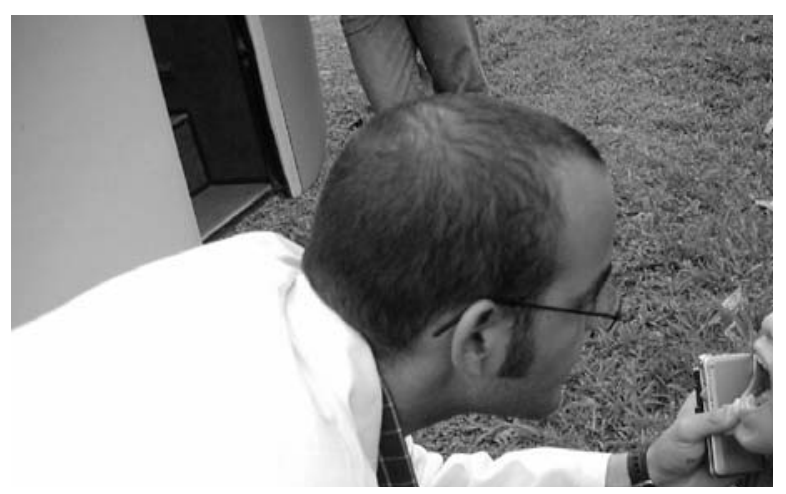

Figure 3. Dental examination

\section{RESULTS}

A total of 49 boys and 39 girls were examined (Figure 4) with the majority of participants being between the ages of 7 and 12. The breakdown of each community is given in Table 1. The average number of serious dental pathologies ranged from 1.6 to about 4 per mouth: 1.66 in La Gamba, 1.82 in Boruca, 3.85 in Maleku, and 3.14 in Bribri (Figure 5). Due to a lack of sample size, Cahuita was omitted from the dental examination

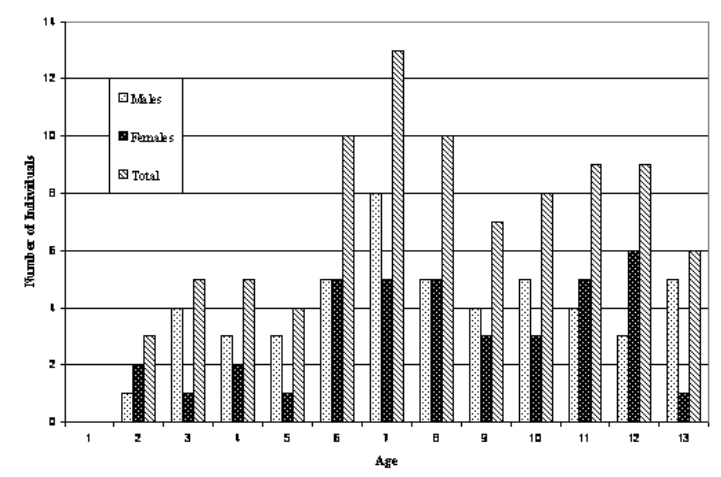

Figure 4. Distribution of sample size

Table 1. Community distributions

\begin{tabular}{lccc}
\hline Community & Av. Dental Path. & Total \# & Male/Female \\
\hline La Gamba & 1.66 & 15 & $9 / 6$ \\
\hline Boruca & 1.82 & 17 & $7 / 10$ \\
\hline Maleku & 3.85 & 28 & $15 / 13$ \\
\hline Bribri & 3.14 & 28 & $18 / 10$ \\
\hline \hline
\end{tabular}

charts; dental hygiene information from interviews, 
however, was still collected from Cahuita.

Pathologies of the anterior teeth (comprised of the incisors and canines) were noted in 34 out of a total of 88 individuals ( $38 \%$ ). The percentage increases to $42 \%$ (31 out of 73 individuals) when data from La Gamba is

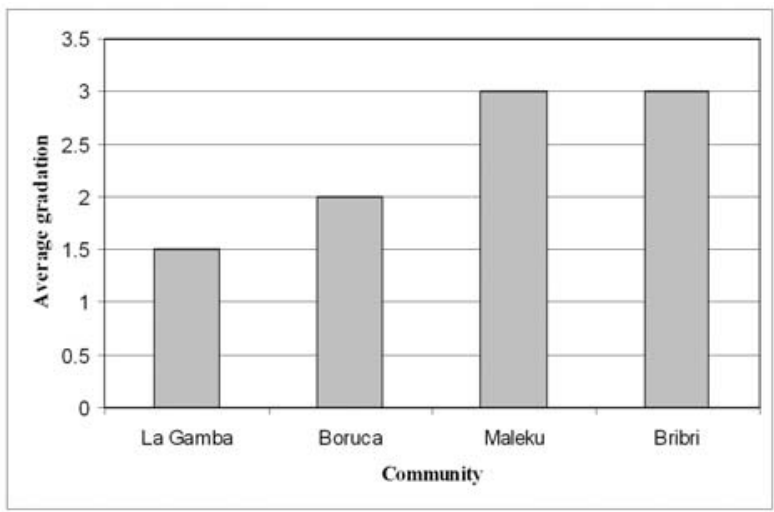

Figure 5. Average number of serious dental pathologies

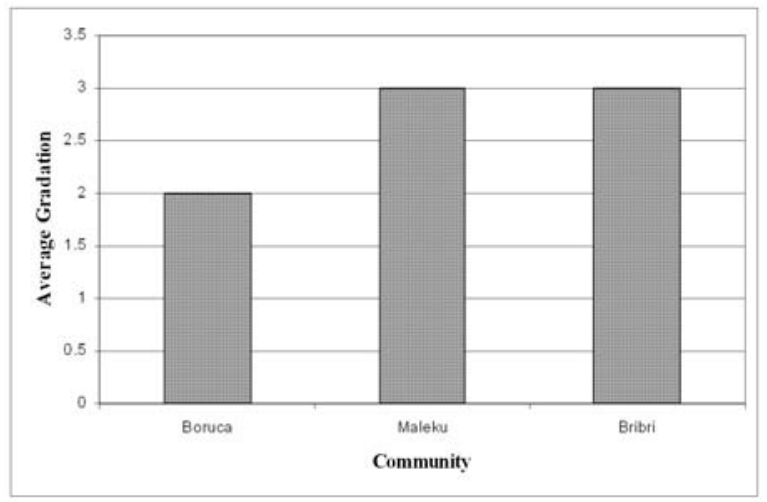

Figure 6. Average number of serious dental pathologies indigenous

excluded, leaving only data obtained from indigenous communities (Figure 6).

Although distinguishable characteristics were observed in each of the five communities, there were also many similarities. Nearly all of the children reported brushing their teeth at least two times per day (76\%). Altogether, 29 of 66 children interviewed (44\%) reported brushing their teeth three times per day. Not all individuals cooperated with all the questions asked of them, therefore only 66 answers were available for the results.

Both adults and children use only a toothbrush and toothpaste to clean their teeth. Colgate is the toothpaste of choice throughout all five communities. Almost all teeth develop cavities and are pulled by the dentist before given the opportunity to fall out on their own. Only three adults reported knowledge of natural remedies for oral conditions. However, these are no longer utilized and none of the children were aware of such treatments. All parents reported brushing their children's teeth from birth through infancy and taking responsibility for teaching them how to brush their own teeth when capable. Most children also receive some form of dental health education at school. The diets among all of the consultants were very similar, consisting mainly of rice, beans, fruits, vegetables, and meat. The most common foods that children associated with being harmful to their teeth were candy, cookies, and chewing gum. The pervasiveness of these saccharines in the diet varied among the communities but overall consumption has increased in recent years.

\section{DISCUSSION}

Consultations with individuals residing in Cahuita and $\mathrm{La} \mathrm{Gamba}$ were used as comparison groups to assist our evaluation of the dental healthcare situation in indigenous communities. Collectively, adherence to oral self-care standards was higher among consultants in Cahuita and La Gamba than in the three indigenous communities. This was indicated by the lower number of average dental pathologies (1.6 in La Gamba vs 1.8, 3.8 , and 3.1). The better oral hygiene practices in Cahuita could be attributed to many factors, such as better school systems, higher income, easily accessible transportation, and the influence of tourists. The construction of the Interamerican Highway near the Boruca community has affected Borucan health in a positive light with the Borucas having considerably less average dental pathologies than the other indigenous communities. The qualitative data from dental examinations further substantiate this large inequity existing between the dental health of indigenous and non-indigenous children of Costa Rica.

The most significant finding was the frequency of anterior pathologies observed (Figures 7-8). Caries lesions are typically found in the posterior dentition where the deep occlusal crenulations (tooth chewing surface morphology) and difficult-to-brush areas are conducive to the formation of cavities. It is uncommon to find caries lesions in the anterior dentition where the tooth morphology is not conducive to residual food and sugar buildups. However, we observed anterior caries in $38 \%$ of the total children studied, and more strikingly, in $42 \%$ of the children in indigenous communities. This becomes even more interesting when one compares this figure to the $44 \%$ of children (all indigenous) who stated that they brushed their teeth 3 times per day.

Yet anterior pathologies are very preventable with simple brushing. They should only arise when food particles are allowed to remain in the interstitial areas for considerable amounts of time. This trend could have arisen from a number of possibilities. The children may 
not be effectively brushing their teeth, or may not be brushing their teeth altogether. Since the numbers given in this document were self-reported, there is the considerable chance that the children over exaggerated their consistency in tooth brushing. Likewise, the amount of saccharines in the diet may directly affect this trend as well. There was a prevalence of eating sticky candies or of chewing sugar cane in all the

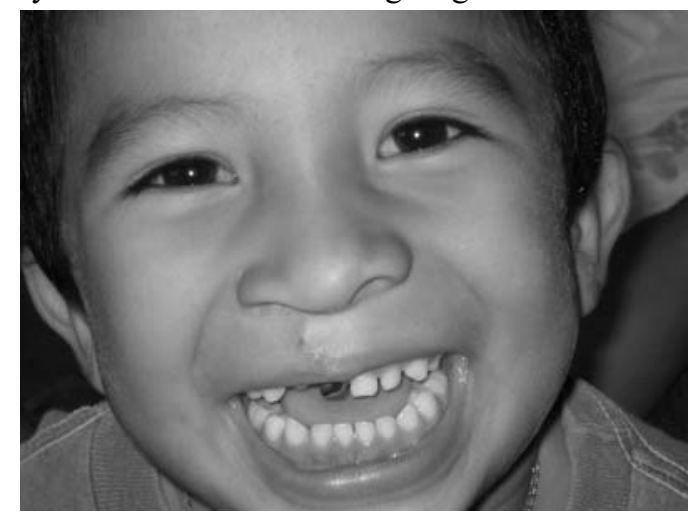

Figure 7. Boruca child, age 4, displaying a case of anterior pathology

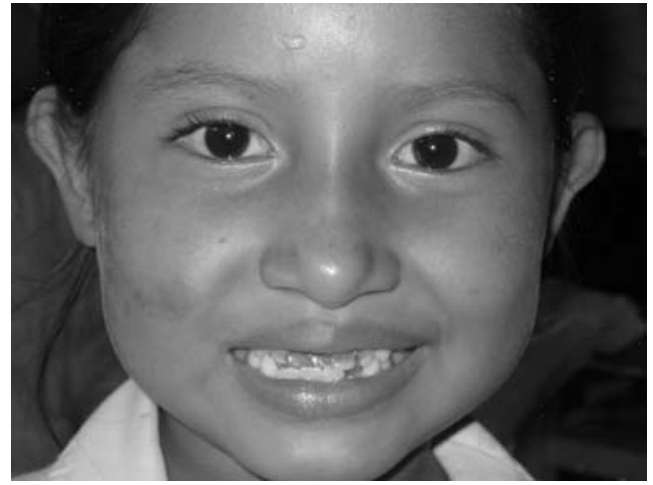

Figure 8. Bribri child, age 7, demonstrating anterior pathology

communities visited, all of which directly contributes to considerable tooth damage without brushing.

At the same time, the severity of other pathologies detected is of considerable importance. Many of the cavities had extended considerably deep within the tooth crown and were completely untreated. In some cases, the cavity succeeded in devouring the tooth crown entirely to the gum line. These results are noteworthy considering the majority of pathologies observed are preventable with simple dental care and attention. With early identification, caries can be treated quickly and easily, thus preventing the catastrophic damage we observed.

Through our comparison of indigenous and nonindigenous communities, it is clear that the governmental resources regarding dental health are not sufficiently reaching the indigenous people. The fact that these communities are isolated significantly impacts their access to dental care. Difficult terrain separates the indigenous communities from urban areas, where better services are often available. And although services are provided within or in close proximity to each of the indigenous reserves, these services are lacking in many areas. First, the clinics are understaffed and there is often only one public dentist to permanently serve an entire indigenous group. Second, the visiting dentists only come to the community sporadically and are not able to visit a significant proportion of the population in the duration of their stay. The lack of adequate dentists is mainly due to unwillingness of nonindigenous dentists to permanently reside in the indigenous communities. Since there are no governmental incentives for a dentist to work near these communities, many choose not to. Additionally, very few, if any, indigenous people attend medical or dental school and therefore cannot bring their expertise back to the community.

Consultants also attributed the cause of poor dental hygiene to a lack of information, education, resources, and help from the government. It should be noted, however, that the extension of governmental services to indigenous communities has only occurred within the past decade. Therefore, it is quite possible that these services provided are still being fine-tuned. The best solution would be for the government to pinpoint existing weaknesses in dental hygiene and develop a straightforward regimen for all citizens of Costa Rica to follow that would combat these issues. Consistent efforts should also be made to enforce this regimen. Members of the indigenous communities reported that the government currently provides minimal preventative education, and that this instruction is not professional and not delivered with authority. Furthermore, dental education has only been introduced to the indigenous communities within the last few decades, and many parents remain unaware of the importance of maintaining healthy oral hygiene. As a result, children also fail to recognize the consequences. Many consultants mentioned that education would significantly improve dental health. Colgate has sponsored a global dental awareness program entitled Sonrisas Brillantes, Futuros Brillantes for the past 15 years. Pamphlets for parents and worksheets for children depict the proper dental care techniques and practices. However, we were the first to introduce these educational materials to the indigenous families.

Overall, the results obtained through this study demonstrate the effects that all these variables have had on these communities. Although dental health education is considerably less in the indigenous populations, much of this can be attributed to the inadequate access to healthcare itself. As the results from Boruca 
demonstrate, there could be a direct correlation with lower average dental pathologies and the Interamerican Highway. The disregard for dental health in the indigenous communities can also be attributed to the general lack of interest by governmental sources. Although the government provides a dentist for each community, the service is often unsatisfactory and insufficient.

Future studies should look at the dental health of adults to see whether there is a correlation across generations. To improve accuracy, a larger sample size should be used at each community and more detailed observations should be made. It would also be of benefit to visit and interview the dentists treating the members of each community. Interviews with residents of La Gamba concerning oral health practices and dental examinations with children of Cahuita would contribute to a more thorough study. Future studies should also look at the impact of the distribution of toothbrushes on the dental health of children. The dental health of these communities should be monitored for detection of any trends. Producers of dental hygiene products should be encouraged to launch pro bono campaigns in indigenous communities.

\section{ACKNOWLEDGEMENTS}

We would like to thank, first and foremost, the very willing and cooperative children and families who allowed us to peer inside of their mouths and ask questions of them as complete strangers. We would also like to thank Colgate-Palmolive (Costa Rica) S.A. for their generous donation of toothbrushes, toothpastes, and dental educational materials. These articles served as excellent compensation for our consultants and hold the potential to drastically impact the dental health of indigenous communities. A. García thanks Mr. and Mrs. Bruce Babcock for their kind donation of scholarship money that made this trip much more feasible.
Likewise, A García would like to thank Dr. Jill Rhodes for her patience and aid with this paper. Without her guidance and help from overseas, this paper would have never flourished. Finally, we would like to thank the Organization of Tropical Studies for providing this opportunity to study the few remaining indigenous groups of Costa Rica. It has truly been an invaluable learning experience.

\section{REFERENCES}

1. CIA. "The World Factbook." Last updated: 20 July 2006. https://www.cia.gov/cia/ publications/factbook/geos/cs.html. Accessed 5 Aug2006.

2. Organization for Tropical Studies (OTS)/Duke University. Introduction to Field Ethnobiology. Undergraduate Study Abroad Program, Participant Observation. 2006.

3. Ministerio de Agricultura y Ganaderia: Programa de Desarrollo Rural. Plan Nacional de Desarrollo de Las Comunidades Indigenas. 2006.

4. U.S. Department of State. Country Reports on Human Rights Practices. Released by the Bureau of Democracy, Human Rights, and Labor. 2001. http://www.state.gov/g/drl/rls/hrrpt/ 2000/what/746.htm. Accessed 5 Aug 2006.

5. Caja Costarricense de Seguro Social, Area de Salud Coto Brus. Analisis de Situación de Salud. San José, Costa Rica. Centro de Desarrollo Estratégico de información en Salud y Seguridad Social. 2004.

6. C.J. "The Deciduous Dentition." 1998. http://www.forensic dentistryonline.org/tooth_morphology/deciduous_dentition. htm. Accessed 5 Aug 2006.

7. Wahl, Shaina. Beyond the Smiles: A look at the teeth of Indigenous children in Costa Rica. Introduction to Field Ethnobiology Course Book, Organization for Tropical Studies Undergraduate Studies Abroad Program 2005

8. Martin, G.J. Ethnobotany: A Methods Manual. Earthscan; Sterling, VA. 2004.

\begin{abstract}
Alfredo Garcia (B. Sc and B.A. 2008) is a 3rd year student at Duke University majoring in religion and biologcal anthropology and anatomy. His research interests include osteology, paleoanthropology and forensics. He is especially passionate about conducting field work on the Middle Stone Age. He is preparing to write a thesis in religion on the topic of Science and Religion. Alfredo plans to apply to medical school in the near future.
\end{abstract}

Christina M. Guzzo (B. Sc 2007) is a 4th year student at Duke University majoring in biology and minoring in chemistry. She has been working in genetic research as well as clinical research on patient safety at the Duke Hospital. Her research interests include public/global health, health policy, sports medicine, and pediatrics. Christina will be attending the University of Michigan Medical School in the upcoming fall semester. 